EXPOSÉ

\title{
La grotte de la Belle-Roche (Sprimont, Province de Liège) : un gisement paléontologique et archéologique d'exception au Benelux
}

\author{
par Jean-Marie Cordy \\ Chercheur au FNRS \\ Maître de conférences à l'Université de Liège \\ Avec la collaboration de Bruno Bastin, Murielle Demaret-Fairon, \\ Camille Ek, Raoul Geeraerts, Marie-Claire Groessens-Van Dyck, \\ André Ozer, Robert Peuchot, Yves Quinif, \\ Jacques Thorez et Marguerite Ulrix-Closset
}

\section{A. Situation}

La grotte de la Belle-Roche est située à environ $20 \mathrm{~km}$ au sud de Liège, à la limite sud-occidentale de la commune de Sprimont (fig. 1). Plus précisément, elle se trouve sur le versant droit de la vallée de l'Amblève, au sud du village de Fraiture, à environ $2 \mathrm{~km}$ du confluent de l'Amblève et de l'Ourthe, près de la localité de Comblain-au-Pont (fig. 2). Elle apparaît au grand jour suivant plusieurs coupes inscrites dans le front oriental de la carrière dite de la BelleRoche, sur la plate-forme supérieure d'exploitation, à une altitude d'environ $160 \mathrm{~m}$, soit à $60 \mathrm{~m}$ au dessus du niveau actuel de la rivière.

La grotte a été mise au jour par les travaux de carrière. Alors que le gisement a été éventré par l'exploitation industrielle depuis des dizaines d'années et qu'il semble avoir déjà été identifié précédemment par des chercheurs amateurs (Cordy, 1981b), ce n'est qu'en 1980 que la réelle importance du site a été reconnue (Cordy, 1980) et qu'une fouille de sauvetage et des études scientifiques ont été entreprises. 


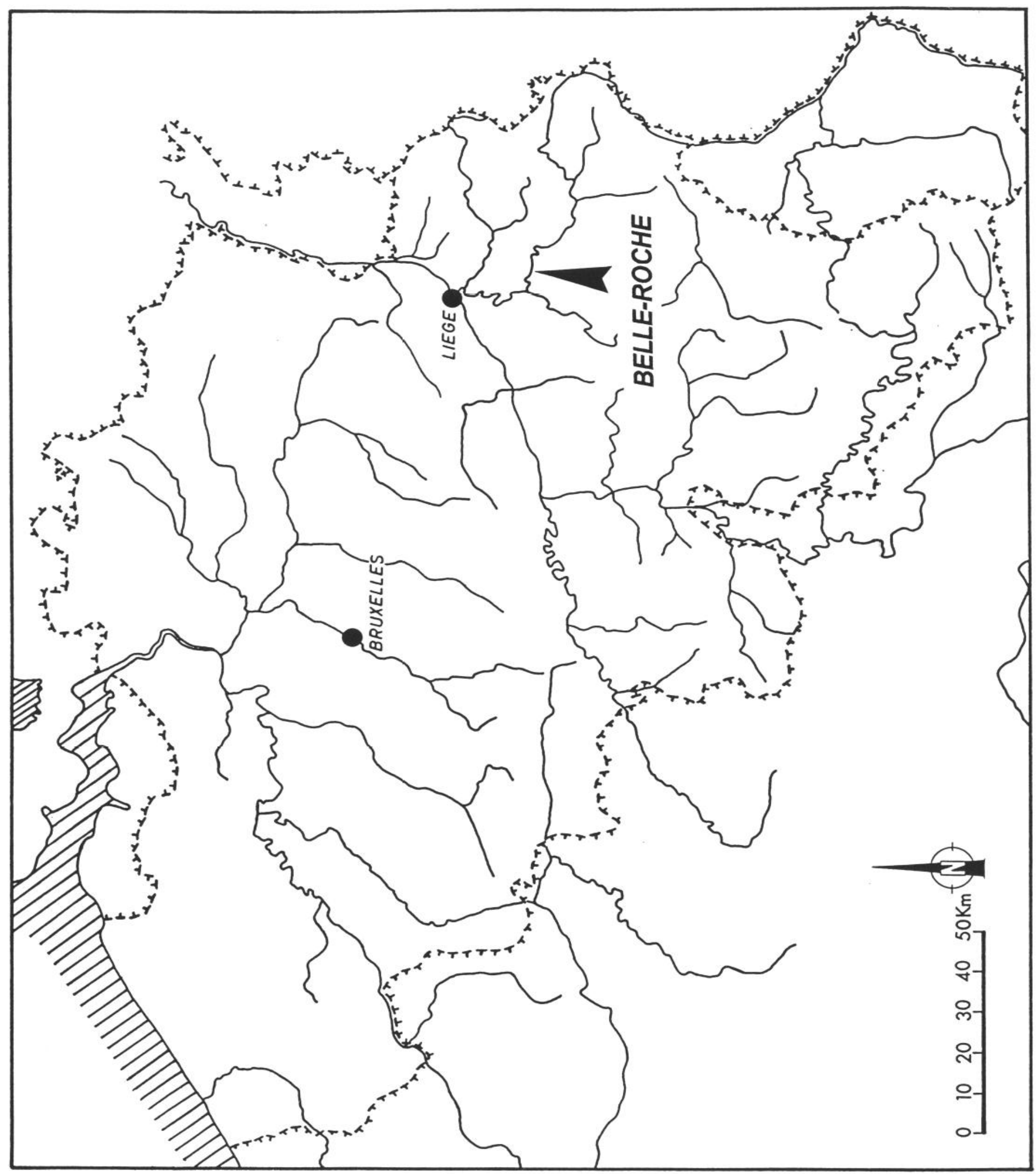

Fig. 1. - Carte de situation du site de la Belle-Roche en Belgique. 


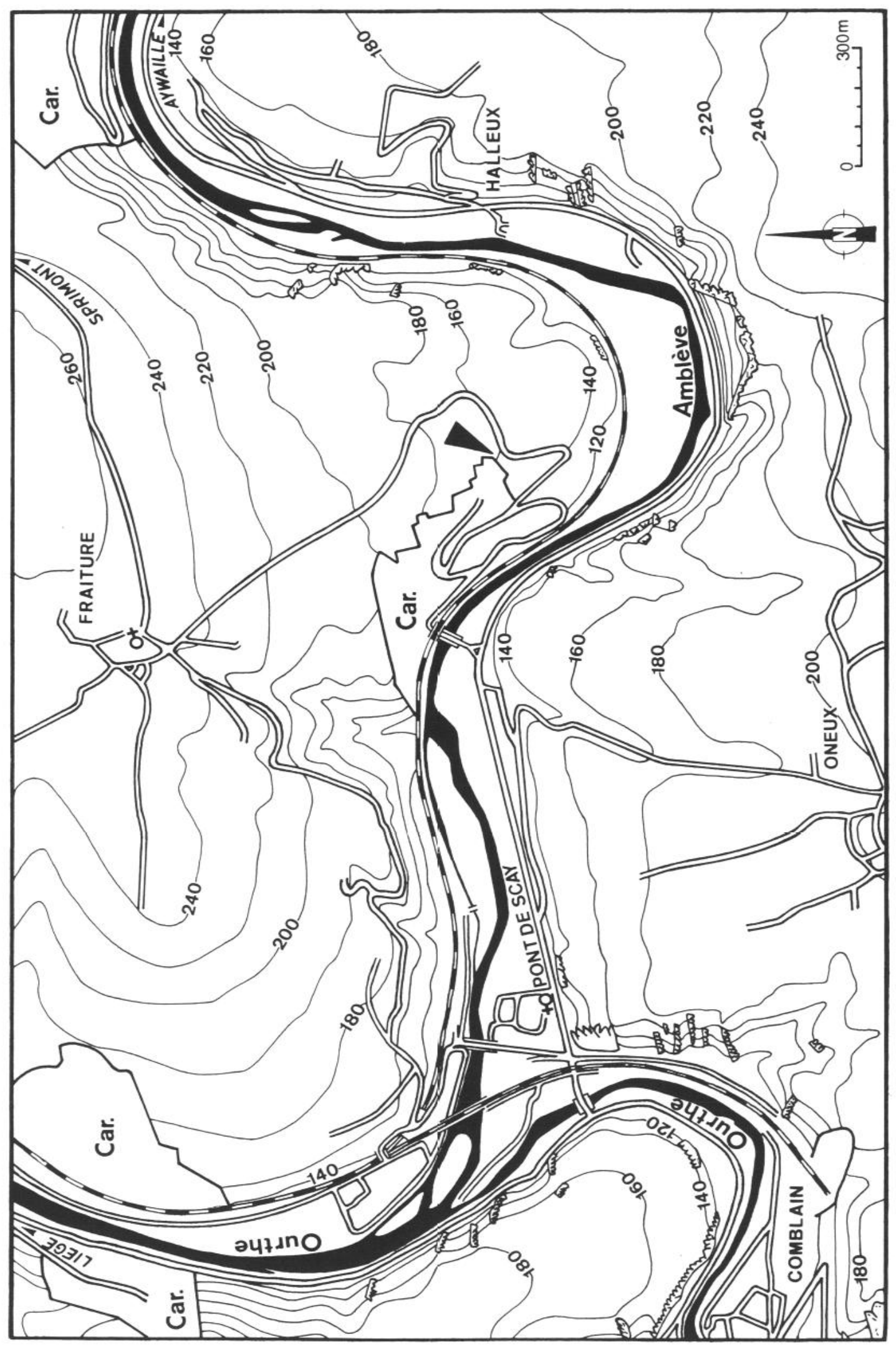

Fig. 2. - Carte détaillée de la localisation de la grotte de la Belle-Roche (sommet du triangle noir) près du confluent de l'Ourthe et de l'Amblève. 


\section{B. Sauvetage du site}

Depuis sa découverte officielle, le gisement de la Belle-Roche a été fouillé par les soins de l'a.s.b.l. «Paléontologie et Archéologie Karstique » en étroite collaboration avec le Service de Paléontologie animale de l'Université de Liège (Prof. G. Ubaghs) et ultérieurement avec l'Unité de Recherche "Évolution des Vertébrés et Évolution humaine " de la même Université. Étant donné la poursuite des fouilles de sauvetage qui sont toujours en cours, seules des publications préliminaires ont été réalisées sur les découvertes et les progrès des recherches (Cordy, 1980, 1981b, 1982, 1987; Cordy et Deuse, 1984 ; Cordy et Ulrix-Closset, 1981 et 1991; Cordy et al., 1992).

Avant la reconnaissance du gisement fossilifère, la portion orientale de la grotte a été détruite sur une longueur difficile à évaluer. Depuis 1980, l'activité de la carrière s'est poursuivie et a continué à détruire progressivement et irrémédiablement la grotte. Dès lors, la fouille a consisté essentiellement à sauver un maximum de données paléontologiques et archéologiques et à recueillir un maximum d'informations à propos du remplissage avant que le site ne soit définitivement détruit par la carrière. Durant les premières années, les recherches ont pu se faire avec toute la précision nécessaire. Cependant, depuis 1987, suite à l'intensification de l'exploitation industrielle, les campagnes de fouille s'étendant sur plus de six mois par année ont été transformées en campagnes de sauvetage. Cette situation était évidemment tout à fait préjudiciable pour la qualité de la fouille proprement dite et pour la récolte des fossiles et des artefacts dont l'état de conservation est assez variable.

Fort heureusement, la carrière a arrêté ses activités depuis 1991. D'autre part, la commune de Sprimont a refusé le permis d'extension de l'extraction et le Ministère de la Région wallonne a pris des mesures de protection temporaire. Le site a été reconnu comme «patrimoine majeur» de la Région wallonne et a été placé sur une liste de sauvegarde, avant d'être loué par la Région pour une période de cinq ans. Cette situation nouvelle permet enfin de travailler sans contraintes et dans des conditions favorables. Outre le développement d'une Ecole internationale de fouilles, les recherches actuelles sont focalisées sur la prospection du gisement dans toute son étendue et sur le sondage de ses potentialités au-delà des coupes fouillées jusqu'à présent. 


\section{Description du site}

Le site correspond à un étage d'un système karstique dont l'orientation générale suit, d'est en ouest, la stratification des bancs de calcaires carbonifères. Différentes coupes transversales et longitudinales mettent au jour quatre galeries qui, parallèles entre elles et globalement horizontales, se prolongent dans la colline encore en place (fig. 3). Associés à ces galeries, des puits et des cheminées karstiques se sont également développés par dissolution différentielle de bancs calcaires dolomitisés.

Le creusement de cet étage karstique a été produit par l'Amblève lorsqu'elle coulait soixante mètres plus haut que son cours actuel et qu'elle recoupait par un cours souterrain un méandre encore visible dans le paysage actuel. Par la suite, cet étage karstique a été abandonné par la rivière qui a creusé la vallée étroite qui la caractérise aujourd'hui. Ensuite, il a été progressivement comblé par des dépôts de remplissage et enfin son aspect extérieur a été profondément modifié par l'érosion quaternaire. Cet étage s'est en quelque sorte "fossilisé » pour devenir un paléokarst impénétrable, sans plus aucune activité hydrologique ou sédimentaire et sans aucun accès visible de l'extérieur.

La largeur transversale des trois galeries fossilifères (II, III et IV), qui sont largement interconnectées, s'étale sur plus de 25 mètres. D'autre part, des sondages géophysiques ont montré que la grotte semble conservée sur une longueur de plus d'une centaine de mètres (ISSEP et Service de Géologie de l'Ingénieur de l'Université de Liège).

\section{Description du remplissage}

Toutes les galeries karstiques sont complètement remplies par des dépôts plus ou moins meubles, certains d'entre eux étant fossilifères. Seul le remplissage de la galerie I doit être distingué des autres par ses caractéristiques sédimentaires; en outre, il s'est révélé stérile tant du point de vue paléontologique que du point de vue archéologique.

Les dépôts des autres galeries se correspondent entre eux en dépit de variations d'épaisseur et parfois de faciès des strates et malgré des déformations locales dues au soutirage karstique au niveau des puits. Cinq phases majeures de remplissage peuvent être définies comme suit (fig. 4 et 5): 


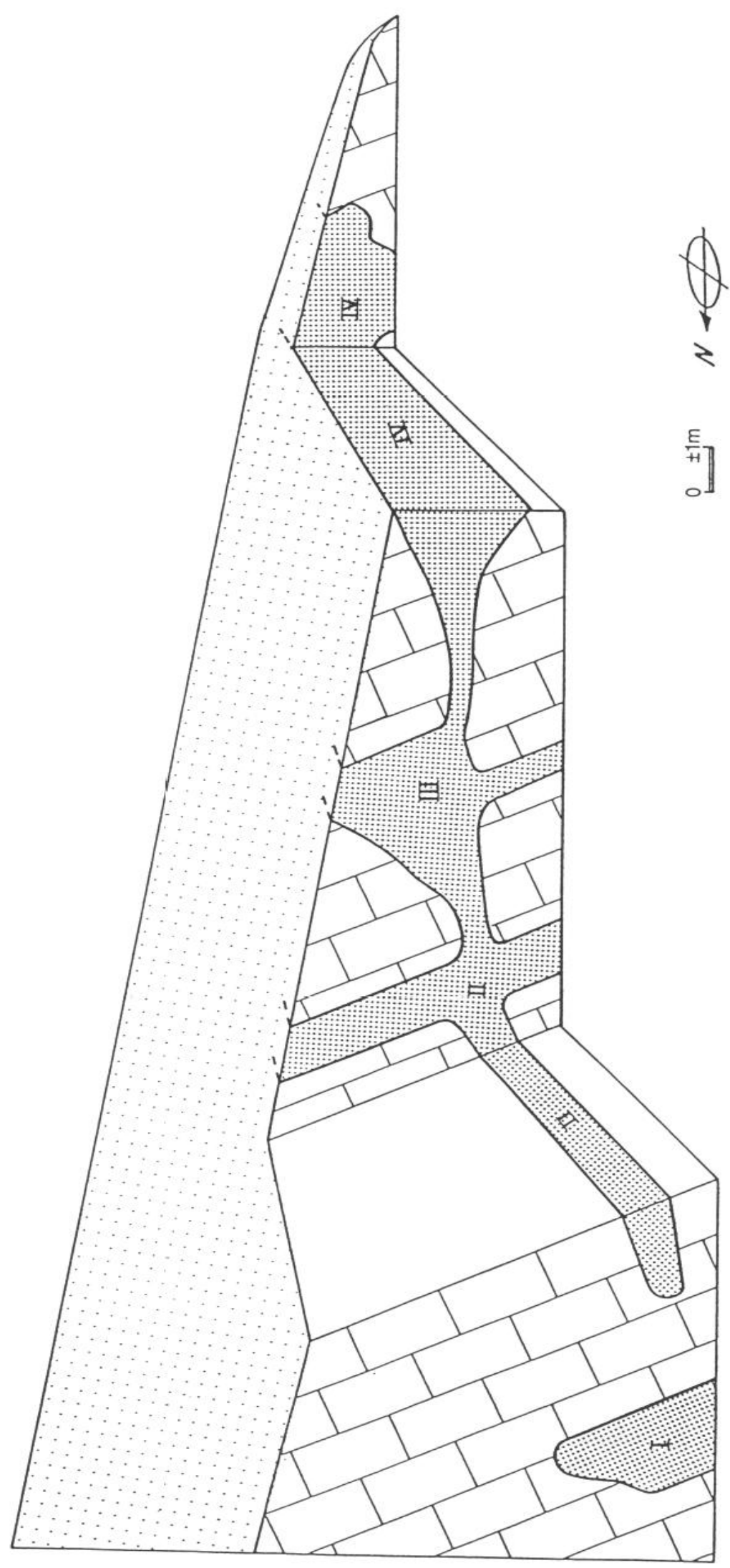

FIG. 3. - Bloc-diagramme montrant schématiquement la disposition des différentes galeries dans le front oriental de la carrière (état initial de 1980). 


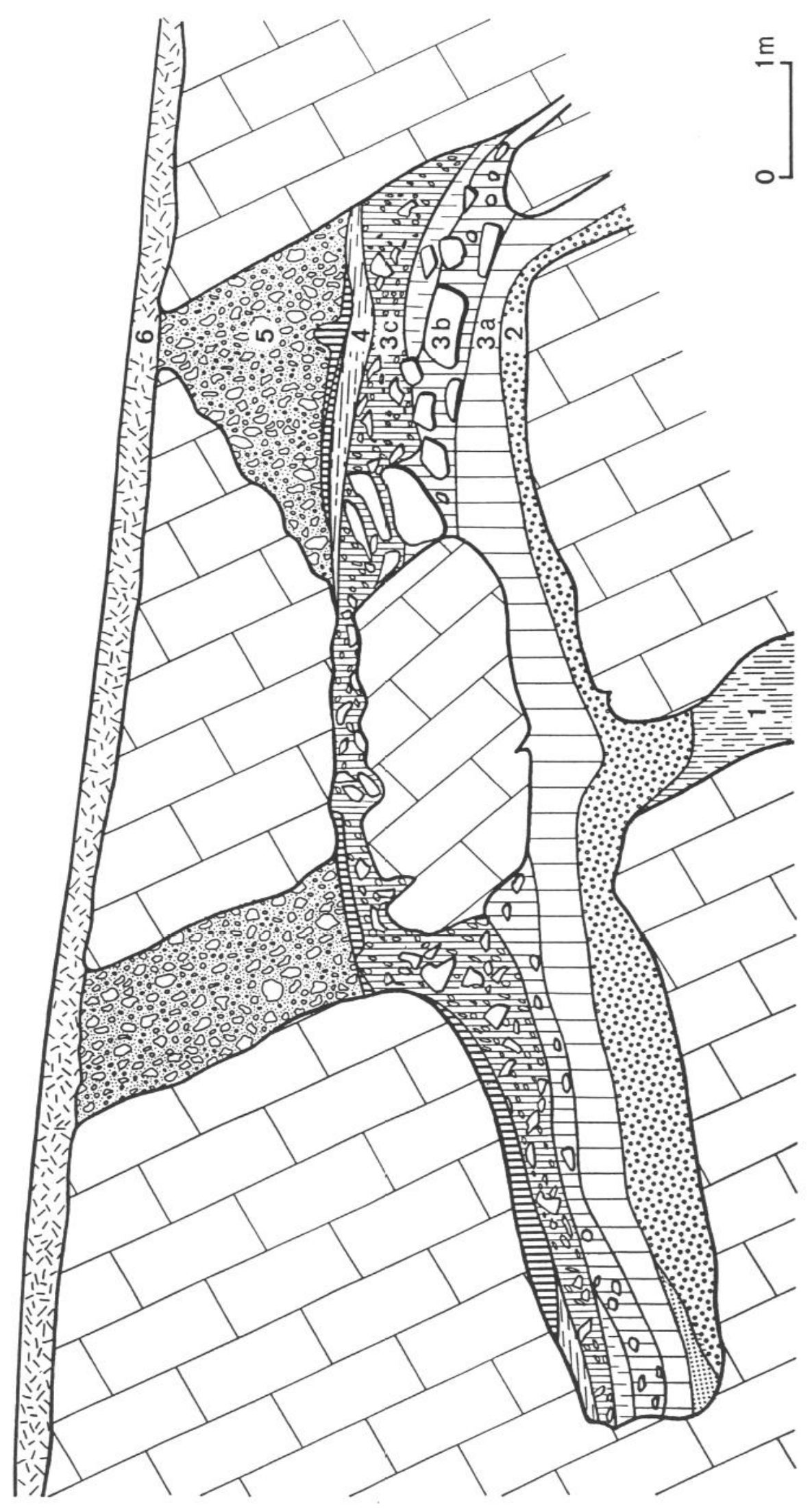

FIG. 4. - Coupe stratigraphique transversale schématique au travers des galeries II et III (voyez les définitions des couches dans le texte). 


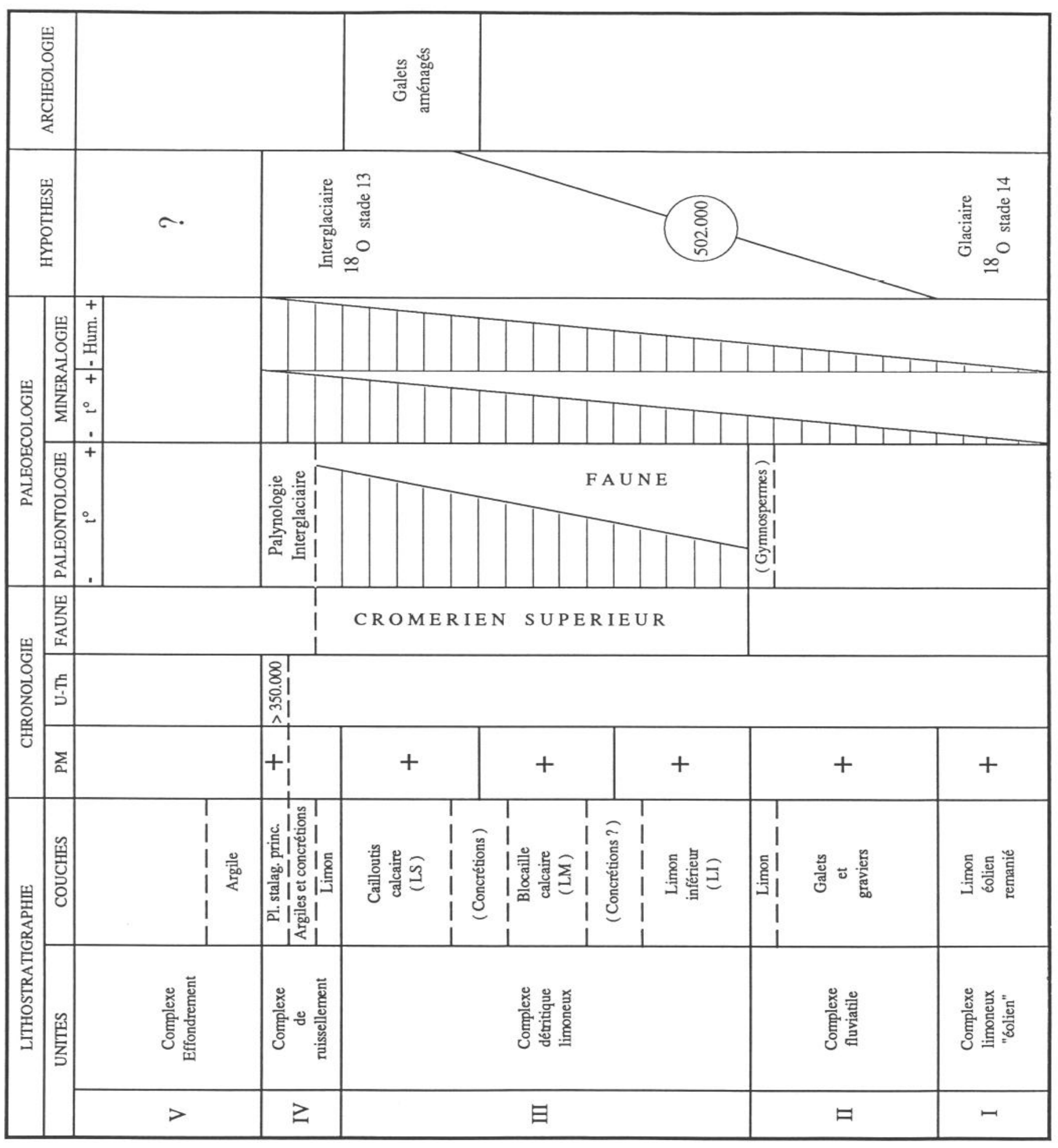

FiG. 5. - Tableau de synthèse des résultats des études pluridisciplinaires. $\mathrm{PM}=$ Paléomagnétisme ; $\mathrm{U}-\mathrm{Th}=$ datation Uranium-Thorium : Minćralogie $=$ minéraux argileux. 


\section{1) Complexe "éolien " de ruissellement :}

Un limon, sans doute d'origine éolienne comme semble l'indiquer le bon classement granulométrique, a comblé initialement les puits karstiques. Le sédiment a probablement pénétré ultérieurement à l'intérieur de la grotte par ruissellement.

\section{2) Complexe fluviatile :}

Le fond des galeries a ensuite été recouvert par un ensemble de couches d'origine fluviatile constituées essentiellement de niveaux de cailloux roulés et de graviers. Compte tenu de la composition lithologique de ces dépôts et de leur disposition, il est clair qu'ils ont été mis en place par l'Amblève lorsque l'activité hydrologique de cet étage karstique était prédominante. A la fin de cette phase, la rivière a encore déposé localement des lentilles de limon sableux, lité et grisâtre. Toutes ces couches sont stériles à l'exception du limon fluviatile qui peut parfois contenir des moulages en limonite de branches de gymnospermes, probablement du genre Pinus (Demaret-Fairon, 1984). Ce complexe forme en quelque sorte une terrasse fluviatile (sensu lato) intrakarstique.

\section{3) Le complexe détritique limoneux :}

Ces dépôts, qui sont très riches en restes fauniques, présentent une matrice limoneuse qui se charge progressivement du bas vers le haut d'argile rougeâtre de décalcification et qui emballe des fragments de calcaire de plus en plus nombreux vers le haut du complexe. Du point de vue sédimentologique, ces dépôts sont granulométriquement mal classés et les cailloux ne présentent pas une orientation bien définie. D'autre part, les restes osseux apparemment dispersés sont rarement en connexion anatomique. Enfin, des concrétions remaniées sont parfois présentes au sein de ces sédiments. Toutes ces observations indiquent une sédimentation complexe avec probablement des phases de remaniements caractérisées en partie par des coulées boueuses et des colmatages de gravité ; il n'est pas impossible que ces remaniements aient déplacé des sédiments qui se trouvaient initialement dans un étage supérieur de la grotte aujourd'hui disparu.

Trois niveaux sont reconnaissables du bas vers le haut:

a) A la base, un limon argileux beige contient des galets épars, similaires à ceux du complexe fluviatile. Ce niveau inférieur contient des restes osseux assez altérés sauf dans la partie supérieure dans laquelle on observe une relative concentration de fossiles qui semble attester un arrêt temporaire de la sédimentation. La présence 
tout à fait sporadique de concrétions au sommet de ce niveau semble également confirmer cette petite discontinuité sédimentaire.

b) Le niveau moyen est constitué par une blocaille calcaire emballée dans une matrice argilo-limoneuse ocre rougeâtre. Dans la galerie II, les blocs de calcaire sont relativement petits et dispersés dans le sédiment meuble; dans les galeries III et IV, cette couche est caractérisée par la présence de très gros blocs d'effondrement et par une blocaille relativement anguleuse. A partir de ce niveau, les fossiles deviennent généralement plus abondants et variés; les restes microfauniques apparaissent et peuvent être localement très abondants. Cette fois encore, mais d'une manière plus évidente, des concrétions stalagmitiques marquent sporadiquement le sommet de ce niveau moyen et attestent un arrêt temporaire de la sédimentation.

c) Le niveau supérieur, qui est caractérisé par une accumulation de galets de calcaire, généralement de taille décimétrique, complète la série détritique. La matrice limoneuse est fort chargée d'une argile rougeâtre qui provient essentiellement de processus de décalcification. L'aspect arrondi de ce cailloutis contraste avec l'aspect angulaire de la blocaille du niveau moyen; des galets de quartz, de quartzite, très rarement de silex peuvent également être observés dans ce niveau. Dans la galerie II, ce cailloutis peut fréquemment être cimenté par une imprégnation calcitique de la matrice sédimentaire et former parfois une brèche tout à fait typique. Ce niveau supérieur est aussi riche en fossiles que le niveau moyen, mais il recèle en plus des artefacts préhistoriques dispersés dans toute son étendue et son épaisseur.

\section{4) Le complexe argilo-calcitique de ruissellement}

Cette unité lithostratigraphique correspond à des processus de ruissellement sur les parois de la grotte, en relation avec un climat très humide. Les sédiments apparaissent sous la forme d'un complexe de couches de calcite, d'argile et de limon argileux, généralement bien litées et stratifiées. Le nombre de couches, leur succession et leur épaisseur, varient selon les endroits de la grotte. Dans la galerie IV, localement, la série commence par une couche limono-argileuse contenant des accumulations d'ossements et de dents de chiroptères. Au sommet de ce complexe, dans les galeries II et III, un plancher stalagmitique peut se développer, atteindre par place une épaisseur d'au moins $15 \mathrm{~cm}$ et servir de base à des stalagmites de tailles diverses. Dans la galerie II, ce complexe argilo-calcitique est quasiment en contact direct avec le plafond de la galerie. Hormis la couche basale riche 
en chiroptères et quelques rares coquilles de gastéropodes disséminées dans ces dépôts, ce complexe est stérile en fossiles et en artefacts.

\section{5) Le complexe d'effondrement}

Dans les parties hautes des galeries III et IV et dans les cheminées karstiques, les cavités restées libres ont été colmatées par les produits de la désagrégation mécanique des parois et du plafond et de l'altération chimique du calcaire (essentiellement du sable dolomitique et de l'argile de décalcification). Ce complexe de couches présente de fréquentes variations de faciès ; néanmoins, il débute typiquement par une couche plus ou moins épaisse d'argile rouge de décalcification qui emballe des fragments épars de concrétions de paroi ou de plafond (stalactites, draperies...).

\section{6) Les colluvions de pente en surface}

En surface, au-dessus du paléokarst, on peut observer des colluvions récentes sur lequelles se développe le sol actuel. Ces colluvions contiennent quelques artefacts mésolithiques et néolithiques. Depuis la formation de la grotte et après son colmatage, le toit calcaire du paléokarst a été de toute évidence considérablement érodé par les processus périglaciaires liés aux glaciations successives. Cette érosion a probablement détruit complètement la partie supérieure de la grotte, peutêtre même un étage du système karstique, dans lequel l'occupation humaine préhistorique a pu éventuellement se produire. L'érosion a été telle que le toit de la grotte correspondant à la galerie IV a disparu en grande partie et que le remplissage de cette galerie est aujourd'hui directement accessible en dessous du sol actuel.

\section{E. Datations}

Le remplissage de cet étage karstique est chronologiquement défini par deux méthodes de datation physique. D'une part, le paléomagnétisme, qui n'a montré qu'une polarité normale dans tous les dépôts, atteste un âge maximal équivalent au début de la dernière période de polarité positive du champ magnétique terrestre, soit environ 730.000 ans (Morley et Hays, 1981 ; Zubakov et Borzenkova, 1990). D'autre part, plusieurs datations radiométriques 234Uranium/230Thorium ont été réalisées par différents laboratoires sur le plancher stalagmitique principal qui recouvre les dépôts fossilifères dans les galeries II et III. Toutes les dates attribuent un âge d'au moins 350.000 ans (limite de la méthode) à cette phase de concrétionnement et a fortiori aux artefacts 
et fossiles qui sont sous-jacents (Gewelt, Gascoyne, Quinif et Cantillana, 1984 ; Gascoyne et Schwarcz, 1985 ; Gewelt, 1985).

L'étude de la faune, qui est exceptionnellement riche et variée (Tableaux 1 à 3), permet d'affiner encore cette fourchette de datation. En effet, l'association et le degré d'évolution des mammifères tels que l'ours de Deninger (Ursus deningeri), le lion primitif des cavernes (Panthera leo fossilis), la panthère de Gombaszög (Panthera gombaszoegensis), le loup de Mosbach (Canis mosbachensis) et une sorte de chien sauvage (Xenocyon lycaonoides), des herbivores tels que le cheval de Mosbach (Equus mosbachensis), le thar (Hemitragus bonali) et le rhinocéros étrusque (Dicerorhinus etruscus), et des rongeurs tels que le campagnol grégaloïde (Pitymys gregaloides) et le campagnol terrestre primitif (Arvicola cantiana), correspondent parfaitement au Pléistocène moyen ancien et plus particulièrement au Cromérien supérieur. Cette faune est géologiquement contemporaine de celles de la grotte de l'Escale (St Estève Janson) en France, de Westbury-sud-Mendip en Grande-Bretagne, des gisements de Mosbach et Mauer en Allemagne et de la grotte d'Atapuerca en Espagne.

Hypothétiquement, la biozone peut être corrélée avec les stades isotopiques océaniques 13 et peut-être 15 (Cordy, 1982, 1992); l'âge des dépôts fossilifères de la Belle-Roche serait dès lors de l'ordre de 500.000 ans \pm 70.000 selon les datations actuellement proposées pour ces stades (Kukla, 1977 ; Morler et Hays, 1981 ; Zubakov et Borzenkova, 1990).

\section{F. Paléoclimat et paléoenvironnement}

D'un point de vue paléontologique, la faune et particulièrement la microfaune indiquent clairement que les dépôts détritiques fossilifères (complexe 3) correspondent à une période de transition paléoclimatique. Dans la partie inférieure des dépôts (niveaux inférieur et moyen), le climat apparaît encore froid et plutôt continental, sans doute en relation avec une période glaciaire. La présence de rongeurs boréaux, tels que le lemming à collier (Dicrostonyx sp.) et le grand lemming (Lemmus cf. lemmus), et celle d'un lemming steppique (Laguriné) et du lièvre des steppes (Ochotona cf. pusilla) typiquement continentaux étayent cette interprétation paléoécologique. D'autre part, même si la présence du renne (Rangifer tarandus) est attestée par un seul fragment de bois, elle confirme le modèle. Dans le haut du remplissage (niveau supérieur), la disparition des espèces précitées et l'apparition d'espèces typiquement sylvicoles, comme le cerf acoronate (Cervus elaphus cf. 
acoronatus) et le chevreuil primitif (Capreolus capreolus cf. sussenbornensis) ou le mulot (Apodemus sp.) parmi les rongeurs, sont des preuves évidentes d'un réchauffement du climat et du développement de biotopes boisés. L'apparition et le développement spectaculaire des chauves-souris surtout au sommet des dépôts fossilifères sous le plancher stalagmitique confirment totalement l'hypothèse de l'avènement d'une période climatique nettement interglaciaire.

Ce modèle paléoclimatique est confirmé par les analyses paléobotaniques, qui apportent quelques indications complémentaires. D'un côté, les analyses polliniques entreprises sur des échantillons de la matrice limoneuse et de la brèche du niveau supérieur du complexe fossilifère (complexe 3), ainsi que sur des échantillons du plancher stalagmitique principal (complexe 4 de ruissellement) fournissent une succession végétale typiquement interglaciaire. Le diagramme pollinique est d'abord caractérisé par la dominance du bouleau (Betula, $48 \%$ ) et du frêne (Fraxinus, $31 \%$ ) et par la présence d'arbres mésophiles tels que le noisetier (Corylus, $3 \%$ ), le charme (Carpinus, $2 \%$ ) et l'aulne (Alnus, 2\%). Il continue ensuite par l'extension combinée du pin (Pinus, $32 \%$ ), du noisetier (Corylus, $12 \%$ ), de l'aulne (Alnus, $11 \%$ ), de l'épicéa (Picea, 9\%), du chêne (Quercus, $1 \%$ ) et du tilleul (Tilia, $1 \%)$. Par la suite, la régression de tous les arbres, exception faite du bouleau (Betula, 12\%) et du chêne (Quercus, $3 \%$ ), semble correspondre à des conditions climatiques plus froides qui restent néanmoins de type interglaciaire comme l'indique la persistance faible mais significative du noisetier (Corylus, 5\%), du frêne (Fraxinus, 1,5\%), du charme (Carpinus), du hêtre (Fagus), du tilleul (Tilia) et de l'orme (Ulmus)(tous quatre moins de $1 \%$ ). D'un autre côté, la fossilisation de branches de gymnospermes (probablement Pinus sp.) (Demaret-Fairon, 1984) peut indiquer que les conditions glaciaires correspondant à la fin des dépôts alluviaux n'étaient pas extrêmes et permettaient le développement d'arbres.

Enfin, l'analyse détaillée des minéraux argileux de l'ensemble des dépôts permet de définir les caractéristiques globales du paléoclimat. Les résultats indiquent qu'il existe deux gradients climatiques au travers de la succession des dépôts de remplissage : un gradient de température allant de conditions périglaciaires (complexe «éolien》1) vers des conditions tout à fait tempérées (complexe 4) et un gradient d'humidité partant de conditions relativement sèches (complexe 1) vers des conditions très humides (complexe 4 de ruissellement). 


\section{G. Paléozoologie}

Les restes osseux et dentaires de mammifères sont particulièrement nombreux : plus de 25.000 pièces ont été répertoriées jusqu'à présent, sans compter les milliers de restes de micromammifères. En plus de la quantité, ce gisement s'individualise avant tout par la variété des espèces présentes. Plus de cinquante espèces différentes de mammifères sont représentées ; elles appartiennent aussi bien à la macrofaune qu'à la microfaune (Tableaux 1 à 3 ). Les carnivores et les rongeurs sont particulièrement bien représentés. A côté des mammifères qui dominent totalement la faune fossile, quelques ossements d'oiseaux ont également été recueillis. D'autre part, le tamisage systématique des sédiments a permis de récolter, outre de nombreux restes de micromammifères, des ossements de reptiles, de batraciens et même de poissons téléostéens. Enfin, une série de coquilles de gastéropodes ont encore été retrouvées à la fouille et au tamisage.

Il s'agit donc d'une faune fossile particulièrement complète, qui permet de définir avec précision la faune mammalienne du Pléistocène moyen ancien dans cette région d'Europe et d'avoir un aperçu de la biocénose dans laquelle elle s'inscrivait. La présence simultanée de grands mammifères et de micromammifères permet aussi de préciser les associations fauniques caractéristiques de cette biozone. En cela, le gisement de la Belle-Roche fournit des informations biochronologiques très utiles à l'échelle européenne, d'autant plus que la faune est bien située dans le temps et le paléoenvironnement par les différentes analyses pluridisciplinaires.

La grande quantité de fossiles récoltés permet également d'entrevoir des analyses populationnelles entre autres sur l'Ursus deningeri (y compris les classes d'âges juvéniles), sur les rongeurs et les chiroptères. Notons encore que certaines espèces peu représentées ou rares dans les sites européens équivalents trouvent d'utiles compléments dans les fossiles récoltés à la Belle-Roche : c'est le cas, par exemple, des restes de grands félins et en particulier de la découverte du premier crâne complet de Panthera gombaszoegensis.

D'un point de vue paléoécologique, une telle accumulation paléontologique tient à plusieurs facteurs : conditions favorables de conservation à l'intérieur de la grotte, lenteur du remplissage karstique, proximité d'accès vers l'extérieur, attraction de la grotte pour les animaux trogloxènes (hôtes temporaires ou occasionnels) (Cordy, 1981a). Concrètement, la grotte servait avant tout de lieu d'hibernation et de partu- 
TABleau I. - Liste des grands mammifères fossiles de la BelleRoche.

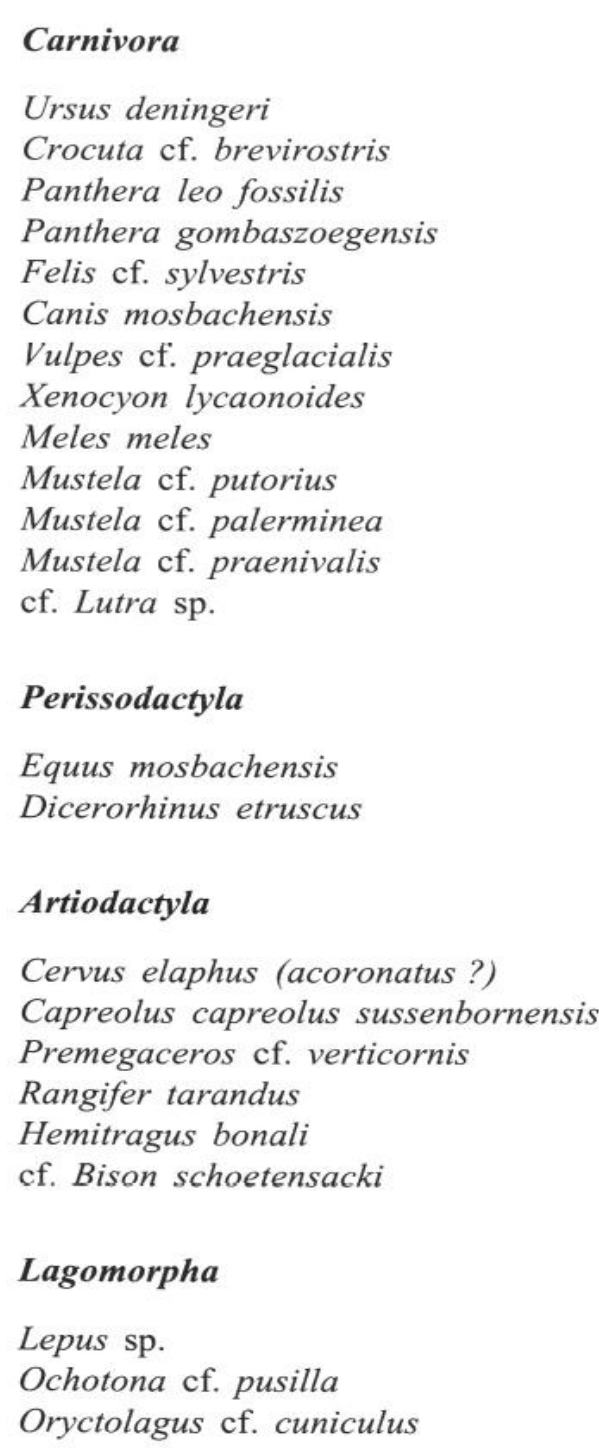

Carnivores
Ours de Deninger
Hyène brévirostre
Lion des cavernes
Panthère de Gombaszög
Chat sauvage
Loup de Mosbach
Renard préglaciaire
Chien sauvage
Blaireau
Putois fossile
Hermine fossile
Belette fossile
Loutre
Périssodactyles
Cheval de Mosbach
Rhinocéros étrusque
Artiodactyles
Cerf acoronate?
Chevreuil de Süssenborn
Prémégacérin
Renne
Thar
Lapièvre des steppes

rition pour l'ours de Deninger dont les ossements et les dents forment approximativement $85 \%$ des restes fossiles. Les autres carnivores ont très certainement utilisé la grotte comme abri ou comme tanière. Tous ces prédateurs qui se sont succédé dans la grotte y ont entraîné les restes de leurs proies; c'est ainsi que les herbivores, les lagomorphes, les rongeurs, les oiseaux sont représentés dans la faune récoltée dans la grotte. Comme prédateurs, il faut encore ajouter, d'une part, l'homme préhistorique et, d'autre part, les rapaces nocturnes qui sont probablement à l'origine de certaines accumulations d'ossements de microvertébrés (restes de pelotes de régurgitation). La présence des chauves- 
TABleau 2. - Liste des micromammifères fossiles de la BelleRoche.

\begin{tabular}{|c|c|}
\hline Rodentia & Rongeurs \\
\hline Eliomys sp. & Lérot \\
\hline Muscardinus sp. & Muscardin \\
\hline Sicista $\mathrm{sp}$ & Siciste \\
\hline Allocricetus bursae & Hamster de Brassö \\
\hline Cricetus cricetus & Grand Hamster \\
\hline Apodemus sp. & Mulot \\
\hline Clethrionomys glareolus & Campagnol roussâtre \\
\hline cf. Pliomys sp. & Campagnol fossile \\
\hline Arvicola cantiana & Grand Campagnol \\
\hline Pitymys gregaloïdes & Campagnol gregaloïde \\
\hline Microtus arvalis & Campagnol des champs \\
\hline Microtus agrestis & Campagnol agreste \\
\hline Microtus malei? & Campagnol de Male \\
\hline Lemmus cf. lemmus & Grand Lemming \\
\hline Dicrostonyx sp. & Lemming à collier \\
\hline Laguriné & Lemming steppique \\
\hline Insectivora & Insectivores \\
\hline Erinaceus sp. & Hérisson \\
\hline Talpa sp. & Taupe \\
\hline Sorex sp. & Musaraignes ( 3 espèces) \\
\hline Crocidura sp. & Crocidure \\
\hline Chiroptera & Chiroptères \\
\hline Myotis bechsteini & Vespertilion de Bechstein \\
\hline Myotis dasycneme & Vespertilion des marais \\
\hline Myotis emarginatus & Vespertilion à oreilles échancrées \\
\hline Myotis nattereri & Vespertilion de Natterer \\
\hline Myotis cf. mystacinus & Vespertilion à moustaches \\
\hline Plecotus cf. auritus & Oreillard \\
\hline
\end{tabular}

souris est tout à fait logique dans le contexte de la grotte qui leur a servi d'abri diurne ou d'abri hivernal. Enfin, il est probable que les cheminées aient pu jouer le rôle de pièges naturels et provoquer l'introduction accidentelle d'animaux dans le milieu karstique; compte tenu de la petite dimension des ouvertures observées jusqu'à présent, ce piégeage n'a pu être effectif que pour de petits animaux.

Soulignons encore que cette faune est sans équivalent au Benelux et que, par sa richesse et sa diversité, elle est exceptionnelle en Europe dans le cadre du Pléistocène moyen ancien. 
Tableau 3. - Liste des animaux fossiles de la Belle-Roche autres que les mammifères.

\begin{tabular}{|c|c|}
\hline Aves & Oiseaux \\
\hline $\begin{array}{l}\text { ? Lyrurus sp. } \\
\text { Passériformes }\end{array}$ & $\begin{array}{l}\text { Petit coq de bruyère } \\
\text { Petit passereau indét. }\end{array}$ \\
\hline Reptilia & Reptiles \\
\hline $\begin{array}{l}\text { Lacertidés } \\
\text { Anguis fragilis } \\
\text { Natrix natrix }\end{array}$ & $\begin{array}{l}\text { Lézard indét. } \\
\text { Orvet } \\
\text { Couleuvre à collier }\end{array}$ \\
\hline Amphibia & Amphibiens \\
\hline $\begin{array}{l}\text { Rana sp. } \\
\text { Anura }\end{array}$ & $\begin{array}{l}\text { Grenouille indét. } \\
\text { Anoure(s) indét. }\end{array}$ \\
\hline Piscii & Poissons \\
\hline Teleostei & Téléostéen(s) indét. \\
\hline Gastropoda & Gastéropodes \\
\hline $\begin{array}{l}\text { Succinea oblonga } \\
\text { Pupilla sp. }\end{array}$ & \\
\hline $\begin{array}{l}\text { Clausiliidés } \\
\text { Discus sp. } \\
\text { Oxychilus cellarius }\end{array}$ & Clausilide indét. \\
\hline $\begin{array}{l}\text { Limacidés } \\
\text { Fruticicola fruticum } \\
\text { Cepaea cf. memoralis }\end{array}$ & Limace indét. \\
\hline
\end{tabular}

\section{H. Industrie préhistorique}

L'industrie préhistorique a essentiellement été recueillie dispersée dans le niveau supérieur du complexe détritique fossilifère (couche $3 \mathrm{c}$ ). Les artefacts n'ont pas été retrouvés rigoureusement in situ, mais ont subi probablement des déplacements limités à l'intérieur de la grotte, suite à des phénomènes de remaniements sédimentaires (coulées de gravité, soutirage karstique...) et biologiques (activités des animaux trogloxènes). Plusieurs descriptions préliminaires des artefacts ont été publiées(Cordy, 1980 ; Cordy et Ulrix-Closset, 1991 ; Ulrix-Closset et Cordy, 1991 ; Cordy et al., 1992).

Les outils lithiques de la Belle-Roche ont essentiellement été fabriqués à partir de galets de silex, caractérisés par un cortex tout à fait usé et de coloration jaunâtre. Les surfaces travaillées et retou- 
chées présentent par contre une patine blanchâtre et sont parfois naturellement un peu émoussées. Sur certaines pièces, des cassures récentes révèlent qu'en dessous de la patine, qui sert de pellicule protectrice, le silex est tout à fait altéré en profondeur. En de rares occasions, des galets de quartz aussi bien que de quartzite ont également été employés. L'origine de la matière première doit être recherchée très probablement dans les dépôts alluviaux de la rivière, sans doute à l'extérieur de la grotte; notons que des galets semblables par leur aspect et leur taille ont été observés dans les dépôts alluviaux de la base du remplissage du paléokarst, ce qui indique que la rivière charriait effectivement à l'époque de tels matériaux.

Le caractère archaïque de l'industrie et son altération imposent une certaine prudence quant à son interprétation. Toutefois, la découverte d'une série d'artefacts présentant des caractéristiques typiques d'une industrie humaine et la découverte de deux petites pièces bifaciales permettent d'assurer sans réserve le caractère anthropique d'une grande part des galets aménagés et des outils sur éclat découverts à la Belle-Roche (fig. 6).

Hormis les deux pièces bifaciales, les artefacts comprennent en priorité des galets aménagés : différents chopping-tools, des galets aménagés atypiques, des nucleus sans préparation et à taille multidirectionnelle et, plus rarement, des choppers. L'industrie se compose également d'éclats parmi lesquels on observe un petit nombre d'outils. Les éclats sont généralement courts et relativement épais, encore qu'il existe quelques rares éclats minces. Le talon ou surface de percussion, corticale ou lisse, forme habituellement un angle ouvert avec la face ventrale d'éclatement; parfois, il est tout à fait réduit ou punctiforme. Les outils sur éclat correspondent avant tout à des racloirs : racloirs simples convexes et une série de racloirs transversaux ; on notera également la présence d'éclats encochés, de quelques outils denticulés et de petits polyèdres.

Parmi les restes osseux recueillis à la Belle-Roche, soulignons encore que deux os d'Ursus deningeri (une phalange et un métapode) présentent des entailles courtes, relativement profondes et angulaires, avec parfois enlèvement de matière (fig. 7). Etant donné la disposition de ces entailles sur l'os, il semble bien qu'il s'agit de traces anthropiques de décarnisation et peut-être de dépiautage de l'animal. 


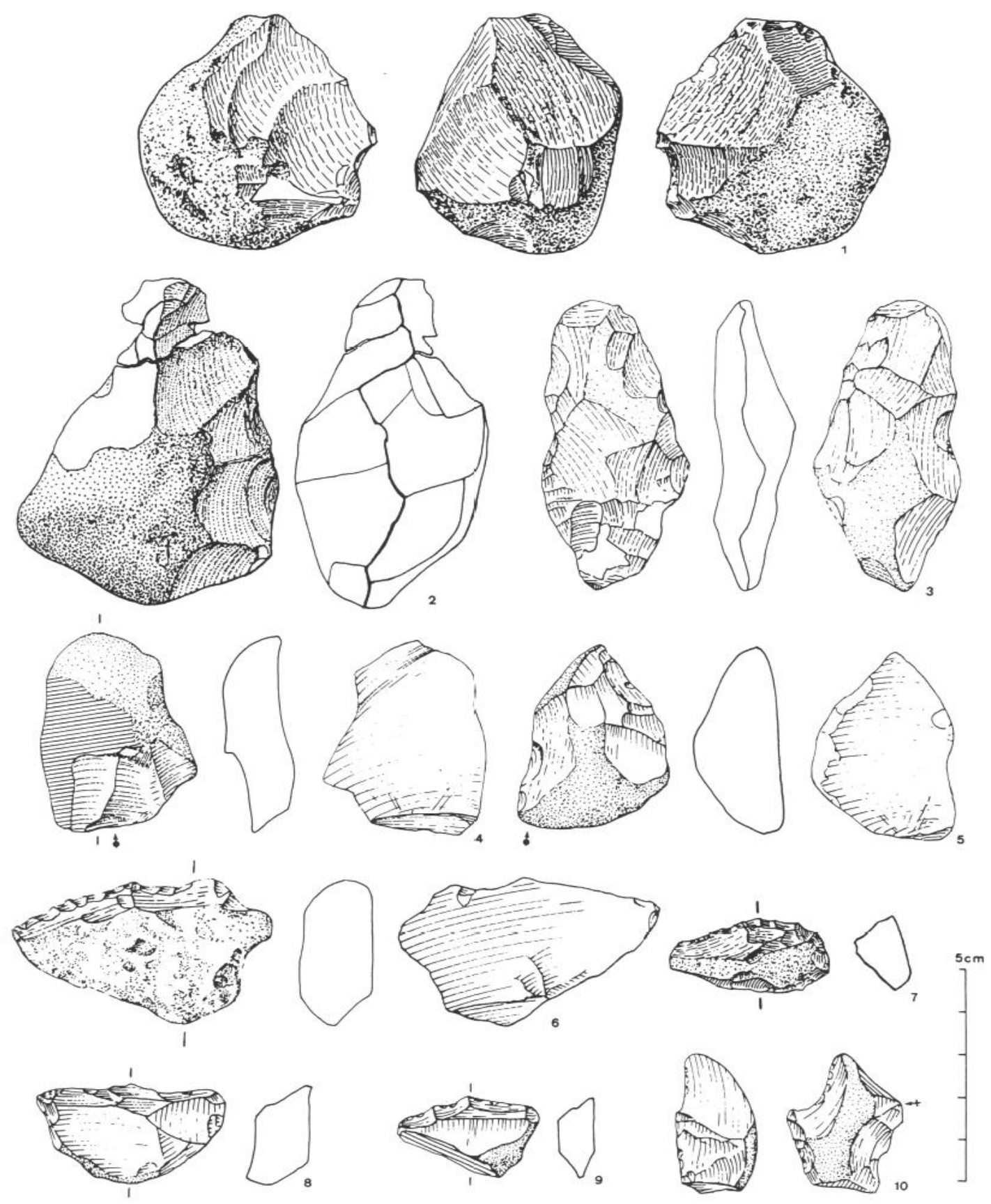

FIG. 6. - Industrie lithique de la Belle-Roche : 1-2, chopping-tools ; 3, outil bifacial ; 4 , éclat ; 5, racloir simple convexe ; 6-8, racloirs transversaux; 9, outil denticulé ; 10 , éclat encoché. 

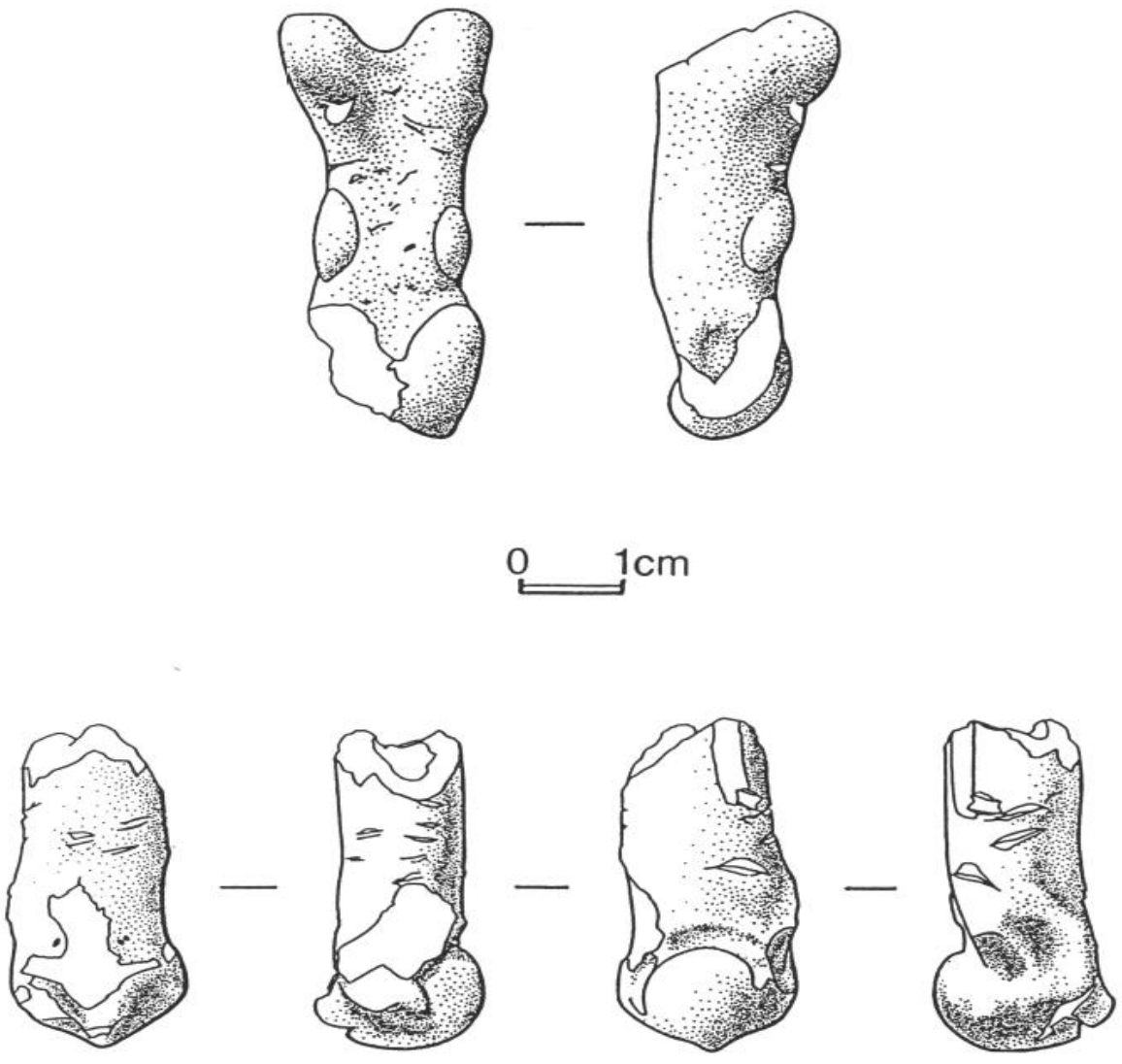

FIG. 7. - Phalange et fragment distal de métapode d'Ours de Deninger avec traces de coups de silex.

\section{J. Sauvegarde de ce patrimoine}

Les recherches multidisciplinaires réalisées dans la grotte de la BelleRoche et l'abondance des données paléontologiques qui y ont été recueillies ont permis d'obtenir une définition chronologique et paléoécologique remarquablement précise du contexte local du Pléistocène moyen ancien et d'une occupation humaine du Paléolithique inférieur. De ce fait, le gisement de la Belle-Roche constitue un site de référence européen et une source d'informations remarquable tant du point de vue de la paléozoologie du Quaternaire que du point de vue archéologique.

Le problème de la conservation de ce site de portée internationale se pose toutefois avec acuité. En effet, un projet d'extension de la carrière, pourrait dans un futur proche, détruire totalement ce patrimoine scientifique et culturel et rendre inopérant tout travail de sauvetage. Seul l'aboutissement de la procédure de classement en cours devrait permettre de sauvegarder ce site paléontologique et archéologique d'exception. En tel cas, la continuité des recherches dans des condi- 
tions optimales à la mesure de l'intérêt de ce gisement serait assurée, de même que le développement de l'Ecole internationale de fouilles préhistoriques fondée sur ce site depuis 1992 par la Région wallonne.

\section{Remerciements}

Depuis la découverte de la grotte en 1980, l'exploitation scientifique du gisement de la Belle-Roche n'aurait pu être menée à bien sans l'aide financière du Ministère de la Communauté française de Belgique, du Ministère de la Région wallonne, du Fonds National de la Recherche Scientifique et sans différents subsides de l'Université de Liège. La commune de Sprimont a également fourni de nombreux services au chantier de fouilles. Enfin, la Région wallonne a encore accordé une série d'emplois subsidiés pour des fouilleurs et des techniciens (CST, TCT, PRIME), tout autant indispensables pour le sauvetage du site. Nous exprimons ici notre profonde gratitude à toutes ces Institutions et à leurs autorités responsables.

Nous adressons également nos vifs remerciements à tous nos collègues qui ont œuvré pour la valorisation scientifique de ce site et aux fouilleurs, professionels ou bénévoles, sans lesquels les fouilles n'auraient pu concrètement être réalisées.

Enfin, nous tenons à remercier le personnel du projet PRIME 10527 pour son aide technique dans la réalisation de cette publication.

\section{BIBLIOGRAPHIE}

CORDY, J.-M., 1980. Le paléokarst de la Belle-Roche (Sprimont, Liège), premier gisement paléontologique et archéologique du Pléistocène moyen ancien en Belgique, C.R. Acad. Sci. Paris, s. D, 291 : pp. 749-751.

CORDY, J.-M., 1981a. Mammifères fossiles et dépôts karstiques, Clair Obscur, 29 : pp. 31-36.

Cordy, J.-M., 1981b. Découverte d'un gisement karstique du Paléolithique inférieur à la carrière de la Belle-Roche, commune de Sprimont, Activ. 80 Serv. S.O.S. Fouilles, 2 : p. 92-98.

CORDY, J.-M., 1982. Biozonation du Quaternaire post-villafranchien continental d'Europe occidentale à partir des grands mammifères, Bull. Soc. Géol. Belg., 105: pp. 303-314.

CORDY, J.-M., 1987. Excursion 6: Le gisement karstique de la Belle-Roche (Sprimont, Province de Liège), Livret-guide Colloq. Int. Sédim. Karst., Hansur-Lesse, 18-22 mai 1987, pp. 55-60.

CORDY, J.-M., 1992. Apport de la paléomammalogie à la paléanthropologie en Europe, in: ToussaINT M., (Éd.), Cinq millions d'années, l'aventure humaine, E.R.A.U.L., 56 : pp. 77-94. 
Cordy, J.-M., Bastin, B., Ek, C., Geeraerts, R., Ozer, A., Quinif, Y., ThoREZ, J. ET Ulrix-Closset, M., 1992. La Belle-Roche (Sprimont, Belgium) : the Oldest Archaeological Site in the Benelux. A report on a Field Trip, in : Toussaint M., (Éd.), Cinq millions d'années, l'aventure humaine, E.R.A.U.L., 56 : pp. 287-301.

Cordy, J.-M. et DeuSE, B., 1984. Un gisement karstique du Paléolithique inférieur à la carrière de la Belle-Roche, commune de Sprimont, in: Decleer, S., (Én.), Spéléologie Lascaux, Catalog. Expo. Mus. R. Art Hist. Brux., pp. 27-32.

Cordy, J.-M. et Ulrix-Closset, M., 1981. La grotte de la Belle-Roche (Prov. de Liège, Belgique) : un gisement à galets aménagés du Pléistocène moyen ancien, Actes $X^{\prime \prime}$ Congr. Union Int. Sc. Préh. et Protohist., Mexico, oct. 1981, Section II, pp. 18-19.

Cordy J.-M. et UlriX-Closset, M., 1991, Synthèse des dernières campagnes de sauvetage au gisement du Paléolithique inférieur de la Belle-Roche (Sprimont), Notae Praehist., 10 : pp. 3-13.

Demaret-Fairon, M., 1984. Végétaux fossiles récoltés dans les paléokarsts de la Belgique, Kölner Geogr. Arb., 45, pp. 107-116.

Gascoyne, M. et Schwarcz, H.P., 1985. Uranium-Series Dates for the Lower Paleolithic Site of Belle-Roche, Belgium, Current Anthropology, 26, pp. 641-642.

Gewelt, M. 1985. Cinétique du concrétionnement dans quelques grottes belges : apport des datations $14 \mathrm{C}$ et $230 \mathrm{Th} / 234 \mathrm{U}$, Ann. Soc. Géol. Belg., 108, pp. 267-273.

Gewelt, M., Gascoyne, M., Quinif, Y. et Cantillana, R., 1984. Les datations radiométriques, Kölner geogr. Arb., 45, pp. 95-104.

Kukla, G. J., 1977. Pleistocene Land-Sea Correlations 1. Europe, EarthScience Review's, 13, pp. 307-374.

Morley, J. J. et HAYS, J. D., 1981. Towards a High-Resolution, Global, Deepsea Chronology for the Last 750,000 Years, Earth and Planet, Sri. I.ett., 53, pp. 279-295.

Ulrix-Closset, M. et CORDY, J.-M., 1991. Les premiers peuplements humains dans le Benelux, in E. BONIFAY et B. VANDERMEERSCH, Les premiers Européens, Paris, Éd. Com. Trav. Hist. et Scient., pp. 177-197.

Zubakov, V. A. et Borzenkova, I. I., 1990. Global Palaeoclimate of the Late Cenozoic, Development in Palaeont. and Stratigr., Elsevier, 12, $456 \mathrm{p}$.

Abstract. This paper provides a synthesis of the multi-disciplinary research which has gone on the La Belle-Roche site (Sprimont, Belgium) for the last thirteen years. This « fossil " cave or palaeokarst has yielded deposits from the Early Middle Pleistocene, a remarkably rich and varied fauna of the Upper Cromerian (around 500.000 years old) and a prehistoric industry involving pebble tools which is by far the oldest traces of human occupation in the Benelux. 